\title{
Endoscopic Arytenoid Abduction Lateropexy for the Treatment of Bilateral Vocal Cord Paralysis in Early Childhood: Case Report
}

\author{
Balázs Sztanó*, Ádám Bach, Vera Matievics, Eszter Erdélyi and László Rovó \\ Department of Otorhinolaryngology and Head and Neck Surgery, University of Szeged, Hungary
}

\begin{abstract}
Objectives: The treatment of bilateral vocal cord paralysis in early childhood is really challenging even now a days. One of the treatment options is endoscopic arytenoid abduction lateropexy; a reversible simple suture vocal cord lateralizing technique, the arytenoid cartilage is directly lateralized to normal abducted position. Authors introduce a case of a successfully treated toddler.

Methods: The 2.5 years old boy had noisy breathing and dyspnea on exertion since delivery. Laryngo-tracheoscopy revealed bilateral vocal cord paralysis. Unilateral, left-sided endoscopic arytenoid abduction lateropexy was performed with supraglottic jet ventilation.

Results: He remained intubated for 3 with an uncuffed tracheal tube. After extubation, no dyspnea or swallowing disorder occurred. During the follow-up laryngo-tracheoscopies, clinical growth charts and voice analysis showed satisfactory functional results.

Conclusions: The endoscopic arytenoid abduction lateropexy is a minimally invasive, quick, reversible endoscopic glottis enlarging procedure, which might be a favorable solution for neonatal bilateral vocal cord paralysis even in early childhood. It provides immediately wide airway without any permanent damage to voice production.
\end{abstract}

OPEN ACCESS

${ }^{*}$ Correspondence:

Balázs Sztanó, Department of Otorhinolaryngology and Head and Neck Surgery, University of Szeged, 111. Tisza Lajos krt, 6725, Szeged,

Hungary, Tel: 3662545310;

E-mail: sztano.balazs@med.u-szeged.

Received Date: 29 May 2018

Accepted Date: 13 Jun 2018

Published Date: 18 Jun 2018

Citation:

Sztanó B, Bach Á, Matievics V, Erdélyi

E, Rovó L. Endoscopic Arytenoid

Abduction Lateropexy for the Treatment of Bilateral Vocal Cord Paralysis in Early Childhood: Case Report. Am J Otolaryngol Head Neck Surg. 2018;

1(3): 1011.

Copyright @ 2018 Balázs Sztanó. This is an open access article distributed under the Creative Commons Attribution

License, which permits unrestricted use, distribution, and reproduction in any medium, provided the original work is properly cited.
Keywords: Bilateral vocal fold paralysis; Dyspnea; Endolaryngeal thread guide instrument; Endoscopic arytenoid abduction lateropexy

\section{Introduction}

The treatment of bilateral vocal cord paralysis is a real challenge especially in early childhood. Associated strider and respiratory distress often require urgent interventions [1-3]. The Endoscopic Arytenoid Abduction Lateropexy (EAAL) is a really effective totally reversible, non-resection 'simple suture' glottis enlarging procedure. Our recent publications objectively proved its efficacy [4-7]. Now, this concept is introduced in a really young patient.

\section{Materials and Methods}

Surgical technique: The Endoscopic Arytenoid Abduction Lateropexy (EAAL) was performed as described in our earlier publications (Figure 1A) [4,8]. Unilateral, left sided lateralization of the arytenoid cartilage was done under general anesthesia via total intravenous anesthesia and high frequency supraglottic jet ventilation with continuous, strict monitoring of saturation and endtidal $\mathrm{CO}_{2}\left(\mathrm{ETCO}_{2}\right)$ and readiness for possible intubation. Jet ventilation was performed by using the Acutronic Ams 1000 device (Acutronic Medical Systems AG, Hirzel, Switzerland) with the following parameters: inhalation time: $40 \%$ to $50 \%$; frequency: $50 /$ minutes to $60 /$ minutes; volume: $200 \mathrm{ml}$ to $300 \mathrm{ml}$; respiratory minute volume 1.2 to 1.51 ; pressure: 50 mbar. The procedure began with direct endoscopic examination of the upper and lower airways to the level of the distal trachea with a rigid $0^{\circ}$ and $30^{\circ}$ endoscope. Bilateral vocal cord paralysis was diagnosed, so performance of EAAL was followed immediately afterwards during the same session.

After disinfection of the laryngeal mucosa, the Endolaryngeal Thread Guide Instrument (ETGI) was led through the laryngoscope to the glottic level (Figure 1B) [4]. The mobile arytenoid cartilage was tilted backward and upward with the end of the instrument. The built-in, curved blade was then pushed through, under the vocal process, and out to the surface of the neck. A non absorbable suture thread (0-Prolene; Ethicon, Somerville, NJ) was laced through the hole at the tip of the blade 


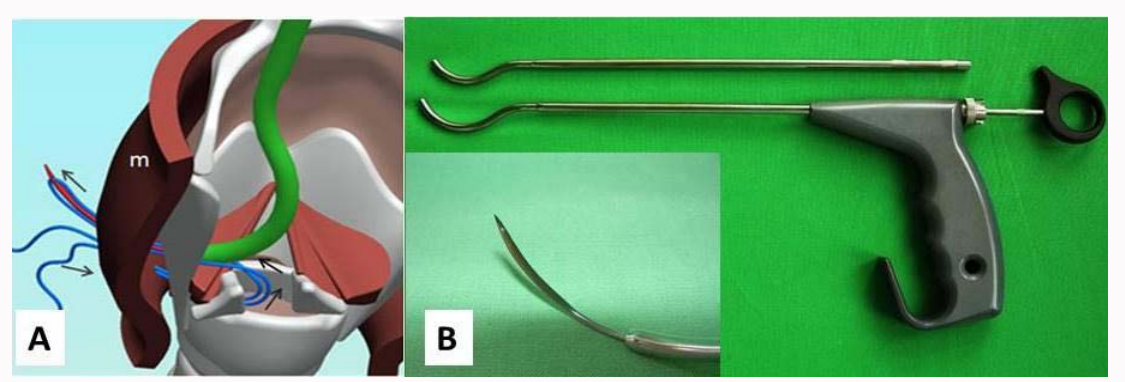

Figure 1: Endoscopic Arytenoid Abduction Lateropexy (EAAL) performed by Endolaryngeal Thread Guide Instrument (ETGI). A: Schematic picture of the procedure: left side arytenoid abduction lateropexy. B: ETGI-with different size stem-pipes and its blade.

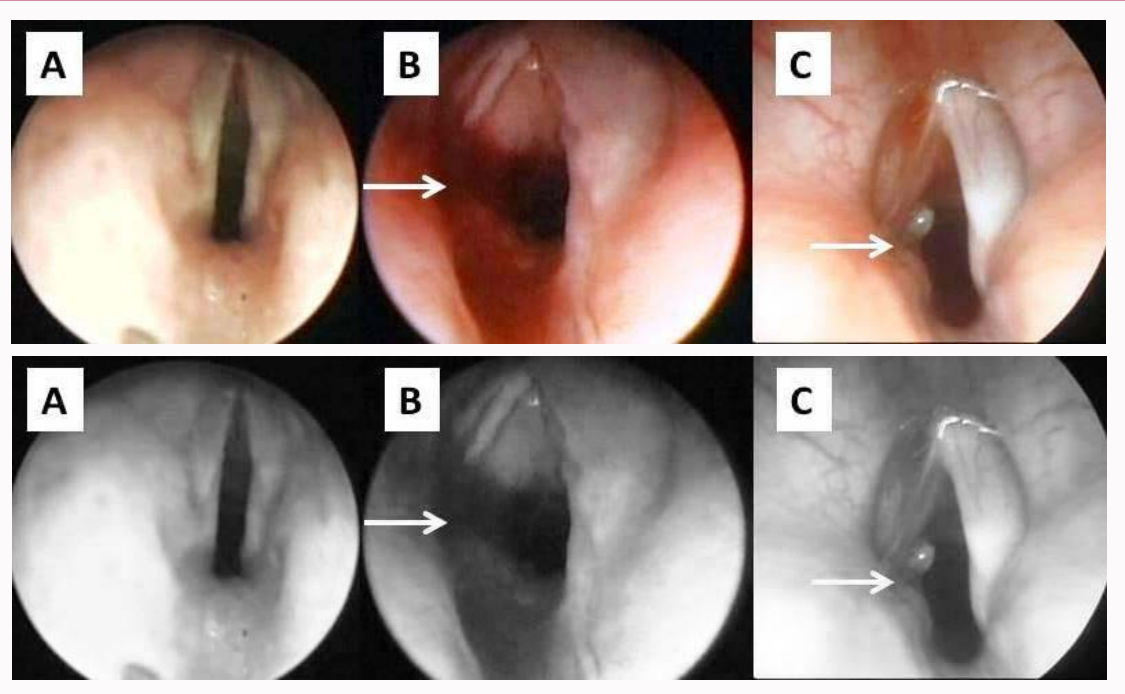

Figure 2: Endoscopic pictures of the lateralized left vocal cord.

A: Intraoperative picture: both vocal cords in paramedian position.

B: Postoperative picture: left side endoscopic arytenoid lateropexy.

C: Late postoperative picture (Postop. $1^{\text {st }}$ year): left vocal cord is lateralized, patent airway Arrows show the lateralization suture.

by an assistant surgeon. The doubled-over thread was pulled back with the blade, into the laryngeal cavity. After a repeated tilting of the arytenoid cartilage, the blade was pushed out with the thread above the vocal process to the outer surface of the neck. The assistant surgeon then cut the double-folded thread to remove it from the blade tip. The blade was then pulled back into the laryngeal cavity, and the ETGI could be removed. A small skin incision was then created to withdraw the ends of the thread by a Jansen hook to the surface of the sternohyoid muscle. The corresponding ends were knotted above it.

\section{Case Report}

V.B. was delivered on the $40^{\text {th }}$ gestation week by caesarean section. Birth weight was $3350 \mathrm{~g}$, but Apgar score 2-7-9. Muscle hypotony and left sided pyelectasia were diagnosed. During his first two years his breathing was always noisy, he had severe dyspnea on exertion. He had several upper airway inflammations. After the admission of the 2.5 years old boy laryngo-tracheoscopy was performed in total intravenous anaesthesia without relaxation, bilateral vocal cord paralysis was revealed (Figure 2A). Unilateral, left side EAAL was done (Figure 2B). He remained intubated (4.0 cuffed tube) for three days, parenteral antibiotic (amoxicillin/clavulanic acid, $25 \mathrm{mg} / 5 \mathrm{mg} /$ $\mathrm{kg} / 12$ hours) and methylprednisolone $(4 \mathrm{mg} / \mathrm{kg}$ ) was administered for one week. After his successful extubation the dyspnea disappeared and was never noticed again. Follow-up evaluations included regular endoscopic examinations under general anaesthesia (Postoperative $1^{\text {st }}$ week, $3^{\text {rd }}$ month and $1^{\text {st }}$ year): lateralized left vocal cords and a right vocal cord in paramedian position were found (Figure 2C). His speech development was normal; the parents were satisfied with the baby's voice. Phoniatry tests at the age of 3.5 years revealed normal Jitter (0.19\%), Shimmer (2.05\%), Harmonics-to-Noise Ratio $(26.54 \mathrm{~Hz})$ and Pitch $(307.68 \mathrm{~Hz})$ values. According to the growth charts the development of the boy was appropriate.

\section{Discussion}

Bilateral vocal cord paralysis in childhood has multiple etiologies including iatrogenic, neurological, idiopathic, joint fixation, birth trauma and idiopathic paralysis. According to the literature, twothirds of the paralytic patients have shown some or even total recovery $[2,9]$. Therefore, many authors recommend 'watch and wait policy', but in some cases the severe dyspnea requires an urgent surgical intervention [10]. The possible treatment options in childhood are much more limited compared to the ones in adulthood. Tracheostomy is still frequently performed $[9,11]$. Due to the high recovery rate the classic irreversible resection procedures (arytenoidectomy and transverse cordotomy) have only limited role $[2,12]$. The optimal surgical treatment would be both reversible and quick, it provides an immediate adequate airway without ruining voice quality and swallowing function. Zawadzka-Głos introduced a simple suture 
lateralization technique of the vocal cord in children aged 1 year and older [13], but this technique did not become popular. Triglia applied the arytenoid lateropexy from an external approach on 15 children between 1 month and 9 years with more encouraging results [14]; however, because of the extensive, surgical dissection of the arytenoid region and the resulting scar formation, this procedure is considered to be irreversible even on adults [15-17].

The Endoscopic Arytenoid Abduction Lateropexy (EAAL) has already been shown to be safe and effective for the treatment of vocal cord immobility of various etiologies $[5,7,8]$. It can be performed in childhood as well; however, the anatomical configuration of the pediatric glottis required a modification of the original Endolaryngeal Thread Guide Instrument (ETGI) [18]. According to our preliminary results the procedure can be relatively easily performed with low surgical stress even in the first days of life [18]. As our case also showed the results are stable and long-lasting. The supraglottic jet ventilation and the novel use of pediatric laryngoscopes ensured excellent visualization of the glottis. Moreover, endoscopic guidance provided the most precise positioning of the lateralization suture. In adult patients, no postoperative intubation, temporary tracheostomy, or intensive care are required after EAAL. Due to the limited pediatric anatomical space, the increased vulnerability, and swelling of the soft tissue a short term postoperative intubation ( 3 days to 5 days) is always prudent along with parenteral steroid and perioperative empiric intravenous antibiotic therapy. After EAAL no feeding difficulty occurred, this was supported by parental report and by the registered weight gain and length growth. Although objective measurements of voice quality are limited at this age, but the results of the voice analysis were appropriate.

Our patient had had dyspnea on exertion before EAAL, which totally disappeared in the postoperative period, so patient's quality of life improved significantly.

\section{Conclusion}

The minimally invasive Endoscopic Arytenoid Abduction Lateropexy (EAAL) might be an effective solution for Bilateral Vocal Cord Paralysis (BVCP) even in early childhood. In one step, the patent airway can be achieved without any permanent damage to voicing or swallowing function. The results seemed to be stable and long-lasting.

\section{References}

1. Benjamin JR, Goldberg RN, Malcolm WF. Neonatal vocal cord paralysis. Neo Reviews. 2009;10(10):494-501.

2. Takamatsu I. Bilateral vocal cord paralysis in children. Nihon Jibiinkoka Gakkai Kaiho. 1996;99(1):91-102.

3. Gentile RD, Miller RH, Woodson GE. Vocal cord paralysis in children 1 year of age and younger. Ann Otol Rhinol Laryngol. 1986;95(6):622-5.
4. Rovó L, Madani S, Sztanó B, Majoros V, Smehák G, Szakács L, et al. A new thread guide instrument for endoscopic arytenoid lateropexy. Laryngoscope. 2010;120(10):2002-7.

5. Sztanó B, Szakács L, Madani S, Tóth F, Bere Z, Castellanos PF, et al. Comparison of endoscopic techniques designed for posterior glottic stenosis-A cadaver morphometric study. Laryngoscope. 2014;124:705-10.

6. Szakács L, Sztanó B, Matievics V, Bere Zs, Bach Á, Castellanos PF, et al. A comparison between transoral glottis-widening techniques for bilateral vocal fold immobility. Laryngoscope. 2015 ;125(11):2522-9.

7. Matievics V, Bach A, Sztano B, Bere Zs, Tobias Z, Castellanos PF, et al. Functional outcomes of endoscopic arytenoid abduction lateropexy for unilateral vocal cord paralysis with dyspnea. Eur Arch Otorhinolaryngol. 2017;274(10):3703-10.

8. Rovó L, Jóri J, Brzózka M, Czigner J. Airway complication after thyroid surgery: minimally invasive management of bilateral recurrent nerve injury. Laryngoscope. 2000;110(1):140-4.

9. Jomah M, Jeffery C, Campbell S, Krajacic A, El-Hakim H. Spontaneous recovery of bilateral congenital idiopathic laryngeal paralysis: Systematic non-meta-analytical review. Int J Pediatr Otorhinolaryngol. 2015;79(2):202-9.

10. Chen EY, Inglis AF Jr. Bilateral vocal cord paralysis in children. Otolaryngol Clin North Am. 2008;41(5):889-901.

11. Miyamoto RC, Parikh SR, Gellad W, Licameli GR. Bilateral congenital vocal cord paralysis: a 16-year institutional review. Otolaryngol Head Neck Surg. 2005;133(2):241-5.

12. Aubry K, Leboulanger N, Harris R, Genty E, Denoyelle F, Garabedian EN. Laser arytenoidectomy in the management of bilateral vocal cord paralysis in children. Int J Pediatr Otorhinolaryngol. 2010;74(5):451-5.

13. Zawadzka-Głos L. Surgical treatment of bilateral vocal cord paralysis in children. New Medicine. 2008;3:70-2.

14. Triglia JM, Belus JF, Nicollas R. Arytenoidopexy for bilateral vocal fold paralysis in young children. The J Laryngol Otol. 1996:110(1):1027-30.

15. Schobel H. Dilatation of the glottis in bilateral vocal cord paralysis. Review of various surgical procedures and a report of personal experience using a functional lateral fixation surgical technic. HNO. 1986;34(12):485-95.

16. Woodson G. Arytenoid abduction for bilateral vocal cord paralysis. Oper Tech Otolayngol Head Neck Surg. 2012;23(3):178-82.

17. Brigger MT, Hartnick CJ. Surgery for pediatric vocal cord paralysis: a meta-analysis. Otolaryngol Head Neck Surg. 2002;126(4):349-55.

18. Madani S, Bach A, Matievics V, Erdelyi E, Sztano B, Szegesdi I, et al. A New Solution for Neonatal Bilateral Vocal Cord Paralysis: Endoscopic Arytenoid Abduction Lateropexy. 2017;127(7):1608-14. 